\title{
An antibiotic-altered microbiota provides fuel for the enteric foe
}

Cell Research (2014) 24:5-6. doi:10.1038/cr.2013.142; published online 29 October 2013

\begin{abstract}
Antibiotic therapies disrupt the intestinal microbiota and render the host susceptible to enteric infections. A recent report by $\mathrm{Ng}$ et al. explores the ability of two intestinal pathogens (Salmonella enterica serovar Typhimurium and Clostridium difficile) to use this disruption to their advantage and consume host carbohydrates that would otherwise be unavailable in the presence of a normal gut microbiota.
\end{abstract}

The human intestinal microbiota is an extremely dense mixed microbial population within the human gut. It is composed of trillions of microbial cells forming a complex and competitive ecosystem that significantly impacts 'host' human cells as well as invading bacterial pathogens [1]. Disruption of gut microbiota diversity with oral antibiotics increases the risk of enteric infections and also causes a number of immune disorders (e.g., atopic dermatitis, asthma, and type 1 diabetes) [2-4]. A recent article by $\mathrm{Ng}$ et al. [5] identifies a mechanism by which invading pathogens exploit this disruption in the intestinal microbiota through the uptake of liberated host carbohydrates. Such a discovery suggests that antibiotics not only negatively affect our indigenous microbiota, but they can also actually promote the proliferation of enteric pathogens.

Enteric pathogens interact extensively with the intestinal microbiota [3], therefore it should be no surprise that these pathogens use an antibioticdisturbed gut microbiota to their advantage. In 2008, Serkirov et al. [6] used increasing doses of vancomycin and streptomycin to disrupt the murine microbiota prior to infecting these mice with $S$. Typhimurium (the causative agent of enteric salmonellosis/gastroenteritis in humans). They found that these antibiotics altered the gut microbiota in a dose-dependent manner. Additionally, in mice treated with increasing doses of antibiotics prior to infection, $S$. Typhimurium was more capable of colonizing the intestinal tract. Stecher et al. [7] found that $S$. Typhimurium actually exploits the host's inflammatory immune response to overcome colonization resistance from the intestinal microbiota. Hence, the mechanisms by which these enteric pathogens evade the protection of our gut microbiota are rather complex and more investigation is required.

In a recent paper published in $\mathrm{Na}$ ture, $\mathrm{Ng}$ and colleagues, based in Justin Sonnenburg's Stanford laboratory, identify a mechanism by which antibiotic-associated pathogens exploit the increase in mucosal carbohydrate availability that occurs after disruption of the gut microbiota with oral antibiotics [5]. Many commensal and pathogenic bacteria use sialic acids acquired from their hosts as an energy source [8]. However, some bacteria, such as Bacteroides thetatiotaomicron (Bt), encode a sialidase required to cleave and release sialic acid from the mucosal glycoconjugates, but lack the catabolic pathway to consume it. $S$. Typhimurium and $C$. difficile conversely possess the nan operon necessary to consume sialic acid within the lumen of the intestine but lack the sialidase to liberate it $[9,10]$.

$\mathrm{Ng}$ et al. infected both germ-free (microbiota-free) and Bt-monoassociated mice with $S$. Typhimurium and $C$. difficile to assess the uptake of free sialic acid by these pathogens in a microbiotadependent environment. In the mice infected with $S$. Typhimurium, transcriptional profiling revealed that the $n a n E$ and fucl operons (genes encoding catabolic pathways for sialic acid and another monosaccharide, fucose, respectively) were significantly upregulated in the $B t$-monoassicated mice relative to the germ-free mice. They constructed a mutant strain of $S$. Typhimurium in which they deleted the nanA and fucl operons. In competition experiments, the mutant strain showed a significant disadvantage in the $B t$-monoassociated mice, yet this deletion had no effect on germ-free mice. C. difficile encodes the nan operon necessary for sialic acid consumption but does not encode any genes related to fucose consumption. $\mathrm{Ng}$ et al. measured the expression of nanA and nanE upon infection of germfree and $B t$-monoassociated mice with C. difficile by quantitative RT-PCR. In $B t$-monoassociated mice both genes exhibited elevated expression levels and an increased density of $C$. difficile relative to germ-free mice. Such findings show that usage of sialic acid by $S$. Typhimurium and $C$. difficile and fucose consumption by $S$. Typhimurium is microbiota-dependent.

The authors also assessed whether sialic acid use is related to pathogen proliferation in antibiotic-treated microbiota. After treatment of conventional 


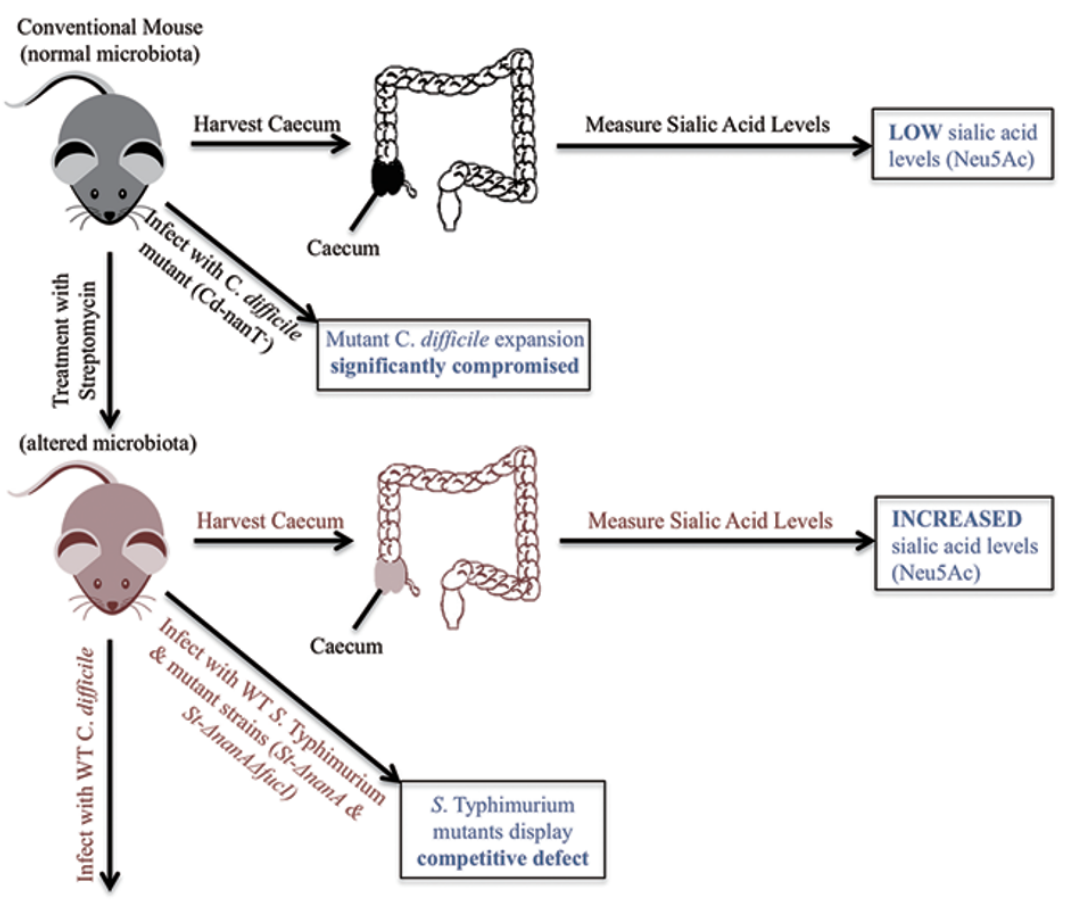

han operon highly induced relative

to C. difficile in vitro model

Figure 1 Schematic overview of antibiotic-altered microbiota experiments. The caecum of conventional mice (normal microbiota) exhibited low sialic acid levels compared to antibiotic-treated mice (altered microbiota). C. difficile mutant expansion was significantly compromised in conventional mice and the $C$. difficile nan operon was highly induced in antibiotic-treated mice. S. Typhimurium mutants displayed a competitive defect in antibiotic-treated mice relative to conventional mice. All results are consistent with the concept that liberated sialic acid allows for the growth and proliferation of WT S. Typhimurium and $C$. difficile within an antibiotic-disturbed gut microbiota.

mice with streptomycin, they quantified free sialic acids in the caeca of these mice and found lower levels of sialic acid in untreated mice relative to the streptomycin-treated mice. $S$. Typhimurium mutants with deletions in the nan and fuc operons (St-AnanA and St- $\Delta$ nanA $A$ fucl) displayed a competitive defect relative to wild-type (WT) $S$. Typhimurium, which is consistent with sialic acid and fucose being necessary for $S$. Typhimurium proliferation. They reported a similar finding in $C$. difficile mutants lacking nanT $\left(\mathrm{Cd}\right.$-nan $\left.T^{-}\right)$, as $C d$-nan $T^{-}$expansion was significantly

relative to conventional mice. Furthermore, the nan operon in WT C. difficile was highly induced in antibiotic-treated mice relative to the $C$. difficile in vitro model. These models support the idea that antibiotics not only disrupt the gut microbiota but they also allow for the release of carbohydrates necessary for the proliferation of enteric pathogens (Figure 1).

Diseases such as vaginal candidiasis, Clostridium difficile colitis, and bacterial urinary tract infections typically arise shortly after antibiotic therapy
[6]. As shown by $\mathrm{Ng}$ et al., it is unlikely that simple disruption of the gut microbiota homeostasis is the cause for these enteric infections. Further insight into the mechanisms involved in antibiotic disruption of the intestinal microbiota is necessary to determine what molecules or mechanisms each of these pathogens is exploiting to effectively grow and proliferate inside its host. Moreover, this study could potentially lead to novel therapeutic strategies, possibly through probiotic regimens using bacteria that target these carbohydrates for digestion or via the administration of drugs that might inhibit the enzymes used by the gut microbiota to free these carbohydrates [5].

\section{Leah T Stiemsma ${ }^{1}$, Stuart E Turvey ${ }^{2}$, Brett B Finlay ${ }^{1}$}

${ }^{1}$ Department of Microbiology \& Immunology, University of British Columbia, Vancouver, BC, V6T 1Z4; ${ }^{2}$ Department of Pediatrics, Child \& Family Research Institute, Vancouver, BC, V5Z $4 H 4$

Correspondence: Brett B Finlay

E-mail: bfinlay@msl.ubc.ca

\section{References}

1 Kau AL, Ahern PP, Griffin NW, et al. Nature 2011; 474:327-336.

2 Penders J, Gerhold K, Stobberingh EE, et al. J Allergy Clin Immunol 2013; 132:601-607.

3 Kamada N, Seo SU, Chen GY, et al. Nat Rev Immunol 2013; 13:321-335.
4 Giongo A, Gano KA, Crabb DB, et al. ISME $J$ 2011; 5:82-91.

$5 \mathrm{Ng} \mathrm{KM}$, Ferreyra JA, Higginbottom SK, et al. Nature 2013; 502:96-99.

6 Sekirov I, Tam NM, Jogova M, et al. Infect Immun 2008; 76:4726-4736.

7 Stecher B, Robbiani R, Walker AW, et al. PLoS Biol 2007; 5:2177-2189.

8 Vimr ER, Kalivoda KA, Deszo EL, et al. Microbiol Mol Biol Rev 2004; 68:132-153.

9 Hoyer LL, Hamilton AC, Steenbergen SM, et al. Mol Microbiol 1992; 6:873-884.

10 Sebaihia M, Wren BW, Mullany P, et al. Nat Genet 2006; 38:779-786. 